\title{
Theory of anomalous corrugation of the Al(111) surface obtained from scanning tunneling microscopy
}

\author{
E. Tekman and S. Ciraci \\ Department of Physics, Bilkent University, Bilkent 06533, Ankara, Turkey
}

(Received 26 January 1990; revised manuscript received 18 April 1990)

\begin{abstract}
We provide an explanation of the observed anomalous corrugation of the $\mathrm{Al}(111)$ surface by calculating the current between the Al(111) sample and tip. An atomically sharp tip images the corrugation of the surface potential, which is enhanced by the tip-induced modifications of the electronic structure. At very small separations the effective barrier due to the lateral confinement of current-carrying states dominates the tunneling, however. This may lead to inversion of the corrugation.
\end{abstract}

Basic theory of scanning tunneling microscopy ${ }^{1}$ (STM) considers the wave functions of the free tip and sample which decay in a potential barrier between the electrodes. The tunneling current was calculated within the firstorder time-dependent perturbation theory by representing the tip apex by a single $s$ wave and is found to be proportional to the local density of states of the free sample $\rho_{s}\left(\mathbf{r}_{0}, E_{F}\right)$ evaluated at the center of the tip and at the Fermi level. ${ }^{2}$ Furthermore, it was shown ${ }^{2}$ that the tunneling current decays exponentially, $I \propto e^{-2 \times d}$, with the distance between the electrodes $d$ and with the inverse decay length given by $\kappa=\sqrt{2 m \phi} / \hbar$. Because of this exponential factor the tunneling current happens to be extremely sensitive to $d$. Assuming that the barrier height $\phi$ is independent of the lateral position of the tip, and also the electronic states of the free sample are not disturbed by the tip, the variation of the measured tunneling current has been related to the variation of $\rho_{s}\left(r_{0}, E_{F}\right)$ of the unperturbed sample.

While several experimental results have been in conformity with the above understanding, some data have been found in serious conflict with it. ${ }^{3}$ For example, the observed STM corrugation of the nominally flat (111) surfaces of the noble ${ }^{4}$ and simple ${ }^{5}$ metals was much larger than one could deduce from the charge density of the free surfaces. Because of these observed anomalous corrugations, attention has been drawn to the tip-sample interaction effects. ${ }^{3}$ Winterlin et al. ${ }^{5}$ argued that the STM corrugation of the $\mathrm{Al}(111)$ surface is enhanced by the elastic deformation of the tip, which is induced by the attractive forces between two electrodes. However, the recent theoretical investigation by Ciraci, Baratoff, and Batra ${ }^{6}$ has been at variance with these arguments. Based on the self-consistent field (SCF) calculations, they showed that the observed corrugation is reduced by the tip-induced elastic deformation, but not enhanced. Moreover, their calculations indicated that the anomalous corrugation has a close bearing on the pronounced changes in the electronic structure of the electrodes. The proximity of the tip induces site-specific and laterally confined states. ${ }^{7,8}$ In particular, a site-dependent effective barrier $\phi_{\text {eff }}$ sets in owing to the lateral confinement of states. ${ }^{6}$ Clearly, the observed anomalous corrugation is a critical problem of STM, and its interpretation by identifying the images is important for a thorough understanding of the three- dimensional (3D) tunneling between the tip and sample.

In this paper, we analyze the variation of the corrugation of the Al(111) surface obtained from STM (Ref. 5) by calculating the current between the tip and sample as a function of lateral and vertical tip positions. We distinguish different ranges of the tip-sample distance based on the different factors which dominate the tunneling current. At large $d \gtrsim 10 \AA$ (distance from ion core to ion core) the STM operates in the independent electrodes regime, thus the corrugation of $\rho_{s}\left(r_{0}, E_{F}\right)$ as well as the corrugation of the potential of the sample surface are negligibly small. However, at relatively smaller $d$ where $\phi$ is reduced but is still finite, the corrugation of the potential at the sample surface near $E_{F}$ is enhanced owing to the tipinduced modifications in the electronic structure. Accordingly, an extremely sharp tip images the width of the potential barrier which is strongly site dependent. In this range the calculated corrugation is $\sim 0.3 \AA$, and is in agreement with the experimentally measured value. ${ }^{5}$ At very small $d, \phi$ collapses, but the site-dependent effective barrier [which is higher at the top $(T)$ site than the hollow $(H)$ site] becomes dominant in tunneling. We predict that in this range of $d(\sim 4 \AA)$ the corrugation is inverted. Upon further approach of the tip a mechanical contact is initiated and the transition from tunneling to ballistic conduction takes place as $\phi_{\text {eff }}$ vanishes. ${ }^{9-11}$

Since the electronic states of the free electrodes are modified by tip-sample interaction ${ }^{6}$ in the experiment, these modifications have to be taken into account in the studies based on the first-order perturbation theory. ${ }^{7}$ This is unfortunately very tedious. In the present study we, however, start with a realistic potential (rather than the electronic states of free electrodes) and obtain the current (or conductance) by evaluating the expectation value of the current operator with respect to the current carrying states calculated from this potential. To this end we model the tip-sample system by using two jellium electrodes separated by a vacuum barrier which depends on the separation of the two jellium edges $l$ [which is smaller than $d$ by the interlayer distance $d_{0}$ between two consecutive (111) atomic planes of $\mathrm{Al}$, i.e., $l=d-d_{0}$ ]. The potential energy between two electrodes can be represented by

$V(l ; z, \rho)=\phi_{m}(l ; z)+\alpha(l ; z) \rho^{2} \theta\left(z+l_{b}\right) \theta\left(l+d_{0} / 2-z\right)$, 
where $\phi_{m}(l ; z)$ is the bimetallic junction potential calculated within the jellium approximation for two jellium edges placed at $z=d_{0} / 2$ (tip) and $z=l+d_{0} / 2$ (sample). In compliance with the SCF calculations ${ }^{6,12} V(l ; \rho, z)$ is parabolic in the transverse plane (i.e., in the $x y$ plane with $\rho^{2}=x^{2}+y^{2}$ ) in the region $-l_{b}<z<l+d_{0} / 2$. A schematic description of the model potential is shown by the inset in Fig. 1. At the tip side $\left(-l_{b} \leq z \leq d_{0} / 2\right)$, $\alpha(l ; z)$ defines the shape of the apex. In the vacuum side $\left(d_{0} / 2<z<l+d_{0} / 2\right)$ the confinement parameters $\alpha(l ; z)$ are obtained from the SCF potential. The jellium parameters of $\mathrm{Al}$ are used for both electrodes (tip and sample). This is, in fact, consistent with the experiment, in which atomic resolution was achieved only after a special treatment ${ }^{4,5}$ providing material transfer from the sample to the apex of the tip.

The current carrying states are the $3 \mathrm{D}$ plane waves in the electrodes and the quantized states in the orifice. Since $\phi_{m}$ and $\alpha$ are varying with $z$, we divide the orifice into discrete segments. In each segment $\phi_{m}(l ; z)$ and $\alpha(l ; z)$ can be assumed constant, so that the wave functions of the eigenstates would be the products of the $2 \mathrm{D}$ isotropic harmonic oscillator solutions and 1D plane waves. Consequently, the current carrying solution $\psi_{\mathbf{k}_{i}}$ corresponding to an incident wave $\mathbf{k}_{i}$ deep in the tip electrode can be written as

$\psi_{\mathbf{k}_{i}}(\rho, z)=\sum_{n}\left[A_{n \mathbf{k}_{i}}(z) e^{i \gamma_{n}(z) z}+B_{n \mathbf{k}_{i}}(z) e^{-i \gamma_{n}(z) z}\right] \Phi_{n}(z, \rho)$,

where $\Phi_{n}(z, \rho)$ is the 2D harmonic oscillator solution for a given $\alpha(l ; z)$ with $n=n_{x}+n_{y}$, and the eigenenergy $\epsilon_{n}(l ; z)=(n+1)\left[2 \hbar^{2} \alpha(l ; z) / m\right]^{1 / 2}$. The propagation constant is given by

$$
\gamma_{n}(z)=\left(\frac{2 m}{\hbar^{2}}\left[E-\phi_{m}(l ; z)-\epsilon_{n}(l ; z)\right]\right)^{1 / 2}
$$

We determine the coefficients $A_{n \mathbf{k}_{i}}$ and $\boldsymbol{B}_{\boldsymbol{n} \mathbf{k}_{i}}$ by using multiple boundary matching. ${ }^{13}$ The total tunneling conductance is obtained by integrating the expectation value of the current operator over the Fermi sphere

$$
\begin{aligned}
G_{t}(l)=\frac{e^{2}}{\pi h} \int_{\mathrm{FS}} \frac{d \mathbf{k}_{\|}}{k_{z}\left(\mathbf{k}_{\|}\right)}\left(\left[\tilde{\mathbf{A}}_{\mathbf{k}}^{\dagger} \operatorname{Re}\{\tilde{\Gamma}\} \tilde{\mathbf{A}}_{\mathbf{k}}-\tilde{\mathbf{B}}_{\mathbf{k}}^{\dagger} \operatorname{Re}\{\tilde{\Gamma}\} \tilde{\mathbf{B}}_{\mathbf{k}}\right]\right. \\
\left.+2 \operatorname{Im}\left[\tilde{\mathbf{A}}_{\mathbf{k}}^{\dagger} \operatorname{Im}\{\tilde{\Gamma}\} \tilde{\mathbf{B}}_{\mathbf{k}}\right]\right),
\end{aligned}
$$

where $\tilde{\mathbf{A}}$ and $\tilde{\mathbf{B}}$ are the vectors of $A_{n \mathbf{k}}$ and $B_{n \mathbf{k}}$, respectively, and $\tilde{\Gamma}$ is the diagonal matrix with $\Gamma_{n n}=\gamma_{n}$. The $z$ dependencies are suppressed since the conductance does not depend on which point the expression in Eq. (4) is evaluated.

Two features in our formalism, namely, the variation of $\phi_{m}(l ; z)$ with lateral position of the tip and the form of $\alpha(l ; z)$ are crucial for the tunneling current and thus relevant for STM corrugation calculated thereof. In what follows we explain how $\phi_{m}$ and $\alpha$ are realistically determined to obtain quantitative results.

It is known that the jellium approximation alone does not convey any information regarding the corrugation of the sample surface, even though it is appropriate to calculate the tunneling current. That is, using only $\phi_{m}(l ; z)$ one may obtain an overall behavior ${ }^{11}$ of tunneling current as a function of $d$, but not its variation with the lateral position of the tip at a given $d$. In order to resolve interactions on the atomic scale an individual atom was attached on one of the jellium surfaces. ${ }^{11}$ Even this approach provides limited applicability in the analysis of the STM corrugation. On the other hand, by using the SCF calculations for the periodically repeating tip-sample system the tipsample interactions can be resolved on the atomic scale and the corrugation of the charge density and potential at the sample surface can be obtained. In this case the calculation of the 3D tunneling current is, however, hindered, since the size of the supercell representing the repeating tip-sample system is finite and thus states in the $k$ space are discretized. In the present study, we combine these two methods. We implement the corrugation of $\phi_{m}(l ; z)$ obtained from the SCF calculations ${ }^{6,12}$ into the jellium model and calculate the tunneling current to infer the STM corrugation.

By using the SCF pseudopotential method the charge density and potential energy of the combined Al tip and Al sample are calculated for different tip positions ( $T$ and $H$ sites) for $d$ ranging from 3.7 to $7.5 \AA$. In these calculations the tip was represented by a pyramid consisting of four atoms, which is attached to the base electrode li.e., an $\mathrm{Al}(111)$ slab]. This pyramidal tip is periodically repeated resulting in a $(3 \times 3)$ tip array. The artificial periodicity is used to represent the wave function by a basis set of $\sim 2000$ plane waves. Since the lateral period is large $(\sim 9 \AA)$, the intertip interaction has no significant effect on the results. We note that the tip-sample system in each periodically repeated supercell is in compliance with our model which represents a single-tip electrode and sample surface as described in Fig. 1. Details of these calculations will be published elsewhere. ${ }^{12}$ Figure 1 illustrates the variation of $\phi_{m}(l ; z)$ obtained from SCF calculations for $l=1.93$ and $3.52 \AA$ (or $d=4.23$ and $5.82 \AA$ ). It is seen that the effective width $\xi(E, d)$ of the potential barrier defined by $\phi_{m}(l ; z)$ at fixed energy is consistently larger at the $H$ site than at the $T$ site. Moreover, the analysis of the calculated $\xi(E, d)$ shows that the corrugation, $\Delta \xi(E, d)=\xi^{H}(E, d)-\xi^{T}(E, d)$, decreases with increasing $d$, and diminish for very large $d$ as anticipated. In the earlier STM studies it was generally assumed that for a fixed $d, \xi(E, d)$ remains constant immaterial of the lateral position of the tip. This way the site-specific variation of the potential barrier has gone unnoticed.

The effect that enhances $\Delta \xi$ at small $d$ can be sought in the tip-sample interaction. Although the surface potential of the free sample $V_{s}(r)$ is dominated by the exchangecorrelation potential (which in the local-density approximation is proportional to $\left.\rho_{s}^{1 / 3}\right) \Delta \xi_{s}(E, h)$ is still a small quantity $(\ll 0.1 \AA)$. This corrugation, $\Delta \xi_{s}(E, h)$, is apparently the measure obtained by $\mathrm{He}$-scattering experiments, $h$ denoting the classical turning point. Only very close to the surface or for energies far below the Fermi level, due to the Coulombic potential (i.e., attractive core and repulsive Hartree potential) the corrugation $\Delta \xi_{s}(E, d)$ is comparatively larger. Nevertheless, these conditions are not accessible with He scattering or with STM operating in the independent (noninteracting) electrode regime. As pointed out earlier, ${ }^{7}$ the tip and sample 


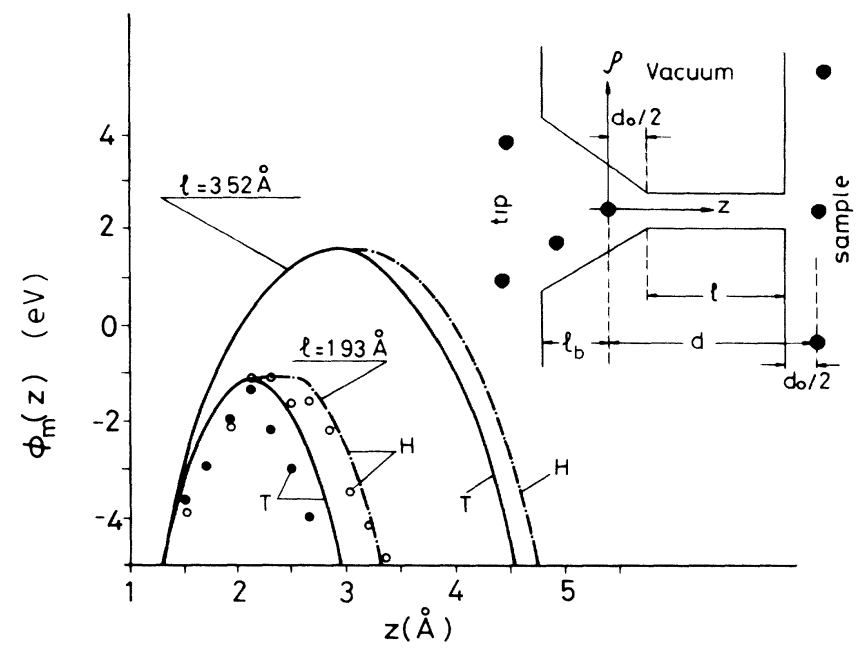

FIG. 1. Variation of $\phi_{m}(l ; z)$ calculated from the jellium model by implementing the corrugation of the corresponding SCF potential. Solid (dashed) lines are jellium results for the top (hollow) site positions of the tip for $l=1.93$ and $3.52 \AA$. The self-consistent results are shown by filled and open circles for the $T$ and $H$ sites, respectively, for $l=1.93 \AA$. Inset shows schematic description of the model used in the calculations of the tunneling current (or conductance). Atomic positions are indicated by the larger filled circles. $d_{0}$ is the interlayer distance of the $\mathrm{Al}(111)$ planes.

states are combined to yield tip induced localized states in STM at small $d$. This induces substantial local modifications in the charge distribution between the two electrodes. Based on the first-principle calculations ${ }^{6}$ it was shown that for $d=4.2 \AA$ the saddle-point value of the charge density of the combined tip-sample system $\rho\left(\mathbf{r}_{\mathrm{sp}}\right)$ is 1 order of magnitude larger than twice the value calculated for the unperturbed sample system at the same point. It is also found that the redistribution of charge is strongly site dependent ${ }^{6,12}$ for an atomically sharp tip. This sitespecific rearrangement of the charge at small $d$ amplifies the corrugation of the charge density $\Delta \rho(d)$, and thus leads to a large value for $\Delta \xi(E, d)$. This important ingredient of the SCF potential is incorporated in the model potential in Eq. (1) in the following manner: First, $\phi_{m}(l ; z)$ is calculated from the jellium approximation for a given $l$, which is in reasonable agreement with the corresponding SCF potential at the $T$ site. Then, $\phi_{m}(l ; z)$ is elongated at the saddle point by $\Delta \xi$ to obtain the potential at the $H$ site. The values of $\Delta \xi$ used in the calculations are listed in Table I.

The form of $\alpha(l ; z)$ determines the lateral confinement of the states between the tip and sample. The larger $\alpha$ (i.e., the steeper the parabolic potential) the stronger the confinement, thus the higher the energies of the subbands $\left[\epsilon_{n}(l ; z)\right]$. In the adiabatic approximation, $\phi_{\mathrm{eff}}(l)$ $=\max \left[\epsilon_{0}(l ; z)+\phi_{m}(l ; z)\right]-E_{F}$ corresponding to a fixed $l$ becomes the effective barrier for an incident wave near $E_{F}$ if $\phi_{\text {eff }}>0$. Consequently, a relatively larger $\alpha$ gives rise to a higher $\phi_{\text {eff }}$ and hence to a smaller tunneling probability. As shown earlier, ${ }^{10}$ because of the lateral confinement the transport occurs via tunneling even if $\phi$ collapses, i.e., even
TABLE I. The parameters used in the calculation of the tunneling current (or conductance). The potential corrugation $(\Delta \xi)$ is calculated from the SCF potential $2.0 \mathrm{eV}$ below the Fermi level. The confinement parameters for the top $\left(\alpha_{T}\right)$ and hollow $\left(\alpha_{H}\right)$ sites are fitted to SCF potential at the bisecting plane.

\begin{tabular}{lccc}
\hline$l(\AA)$ & $\Delta \xi(\AA)$ & $\alpha_{T}\left(\mathrm{eV} / \AA^{2}\right)$ & $\alpha_{H}\left(\mathrm{eV} / \AA^{2}\right)$ \\
\hline 2.0 & 0.325 & 0.459 & 0.328 \\
3.0 & 0.240 & 0.157 & 0.166 \\
4.0 & 0.191 & 0.068 & 0.093 \\
5.0 & 0.158 & 0.034 & 0.056 \\
6.0 & 0.135 & 0.019 & 0.036 \\
\hline \hline
\end{tabular}

$\max \left[\phi_{m}(l ; z)\right]<E_{F}$. As $d$ is approaching the separation corresponding to maximum binding (or zero force) the effective barrier may also collapse (i.e., $\phi_{\mathrm{eff}}<0$ ). In this case, the character of the conductance undergoes a change, and ballistic transport takes place. In the ballistic regime, the conductance can be quantized ${ }^{10,13}$ depending on the lateral and longitudinal extent of the orifice. It becomes clear that the present formalism with realistic $\phi_{m}(l ; z)$ and $\alpha(l ; z)$ allows us to study the transport between the tip and sample in a wide range covering the tunneling and ballistic regimes. Earlier, thorough analyses of the tunneling and "quantized" ballistic regimes in STM were also presented by a similar approach. ${ }^{10,13}$ In Table I, we list the values of $\alpha$ used in the calculations, which are taken to be constant in the region $d_{0} / 2<z<l+d_{0} / 2$.

Having implemented the corrugation $\Delta \xi$ and the correct form of $\alpha(l ; z)$ in Eq. (1), we finally calculate the tunneling conductance $G_{t}$ as a function of $l$. Our results are presented in Fig. 2. For large $l(\gtrsim 5 \AA), \log _{10} G_{t}$ vs $l$ curve is approximately a straight line with a constant negative slope. This indicates that the transport occurs via tunneling. In this range of $l$, the current at the $T$ site is larger than that at the $H$ site and yields corrugation of $\sim 0.3 \AA$. This value is in agreement with the experimental observation, ${ }^{5}$ since the tunneling current is $\sim 10-20$ $\mathrm{nA}$ for $l \sim 5.5 \AA$ and for the bias voltage of $50 \mathrm{mV}$ (which are typical for the observed anomalous corrugation ${ }^{5}$ ). Note that for increasing $l$ the corrugation in Fig. 2

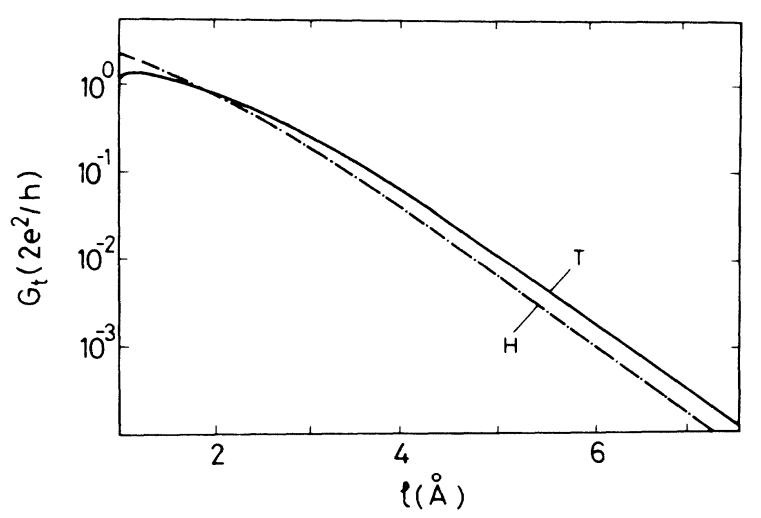

FIG. 2. The tunneling conductance calculated by using Eq. (4). The solid (dash-dotted) curve is for the top (hollow) site. 
remains approximately constant. This is due to an insufficient fit of $\alpha(l)$ to the SCF results. For a more realistic form of $\alpha$, the barrier at the $T$ and $H$ sites should merge into one leading to zero corrugation. In the intermediate region $2 \lesssim l \lesssim 4 \AA$, the effect of increasing lateral confinement (i.e., higher $\phi_{\text {eff }}$ ) at the $T$ site becomes superior to that of increasing $\Delta \xi$ at the $H$ site. Hence, the measured corrugation has to decrease with decreasing $l$, in spite of (and because of) the increasing tip-sample interaction. Finally as shown in Fig. 2, for $l \lesssim 2 \AA$ the current at the $H$ site exceeds that at the $T$ site. This implies that the corrugation is inverted at small $l$ before the mechanical contact, and thus the hollow site (rather than the atomic sites) appears as a protrusion in the STM images obtained by the topographic mode. Note, however, that the inverted corrugation may not be easily observable owing to the mechanical instability of the tip in this regime.

In conclusion, we used a model potential to calculate the tunneling current for an $\mathrm{Al}$ tip and $\mathrm{Al}(111)$ sample system. We have found that the observed anomalous corrugation is related to the corrugation of the potential barrier, which is enhanced by the tip-sample interaction effects. Novel effects, namely, decreasing corrugation with increasing current and inverted corrugation, were predicted.

This work is partially supported by Joint Project Agreement between Bilkent University and IBM Zurich Research Laboratory. We acknowledge stimulating discussions with Professor A. Baratoff, Dr. I. P. Batra, and Dr. R. J. Behm.
${ }^{1}$ G. Binnig, H. Rohrer, Ch. Gerber, and E. Weibel, Phys. Rev. Lett. 49, 57 (1982).

2J. Tersoff and D. R. Hamann, Phys. Rev. Lett. 50, 1398 (1983).

${ }^{3}$ For a review see S. Ciraci, in Basic Concepts and Applications of Scanning Tunneling Microscopy and Related Techniques, edited by R. J. Behm, N. Garcia, and H. Rohrer (Elsevier, Amsterdam, 1990).

${ }^{4}$ V. M. Hallmark, S. Chiang, J. F. Rabolt, J. D. Swalen, and R. J. Wilson, Phys. Rev. Lett. 59, 2879 (1987).

${ }_{5}$ J. Winterlin, J. Wiechers, H. Brune, T. Gritsch, H. Höfer, and R. J. Behm, Phys. Rev. Lett. 62,59 (1989).

${ }^{6}$ S. Ciraci, A. Baratoff, and I. P. Batra, Phys. Rev. B (to be pub- lished).

${ }^{7}$ E. Tekman and S. Ciraci, Phys. Rev. B 40, 10286 (1989).

${ }^{8}$ S. Ciraci, A. Baratoff, and I. P. Batra, Phys. Rev. B 41, 2763 (1990).

9 J. K. Gimzewski and R. Möller, Phys. Rev. B 36, 1284 (1987).

${ }^{10}$ S. Ciraci and E. Tekman, Phys. Rev. B 40, 11696 (1989).

${ }^{11}$ N. D. Lang, Phys. Rev. Lett. 56, 1164 (1986); Phys. Rev. B 36, 8173 (1987); 37, 10395 (1988).

${ }^{12}$ S. Ciraci, E. Tekman, A. Baratoff, and I. P. Batra (unpublished).

${ }^{13}$ E. Tekman and S. Ciraci, Phys. Rev. B 39, 8772 (1989), and references therein. 